Bulletin of Pharmaceutical Sciences
Assiut University
Website: http://bpsa.journals.ekb.eg/
e-mail: bullpharm@aun.edu.eg

\title{
SYNTHESIS, BIOLOGICAL EVALUATION AND IN SILICO ADME PREDICTIONS OF SOME NEW COUMARIN-ACETAMIDE DERIVATIVES AS POTENT ANTI-INFLAMMATORY AGENTS
}

Mohammed A. I. Elbastawesy ${ }^{1}$, , Martha M. Morcoss ${ }^{2}$, Mostafa H. Abdelrahman ${ }^{1}$, Bahaa G.M. Youssif $^{3}$ and Alaa M. Hayallah ${ }^{3,4}$

\author{
${ }^{1}$ Pharmaceutical Organic Chemistry Department, Faculty of Pharmacy, Al-azhar \\ University, Assiut Branch 71524, Assiut, Egypt. \\ ${ }^{2}$ Department of Pharmaceutical Chemistry, Faculty of Pharmacy, Nahda University, \\ 62513 Beni-Suef, Egypt \\ ${ }^{3}$ Pharmaceutical Organic Chemistry Department, Faculty of Pharmacy, Assiut University, \\ Assiut 71526, Assiut, Egypt. \\ ${ }^{4}$ Pharmaceutical Chemistry Department, Faculty of Pharmacy, Sphinx University, New \\ Assiut, Egypt
}

A new series of 4-methyl-7-methoxycoumarin derivatives linked with three amide groups were prepared starting from 2-((4-methyl-2-oxo-2H-chromen-7-yl)oxy) acetohydrazide, compound 4. TLC was used to ensure the purity of all new compounds, and IR, IH NMR, and mass spectrometry, as well as elemental microanalyses, were used to confirm their structures. All of the target compounds were tested for anti-inflammatory activity. The results showed that most of the tested compounds exhibited significant anti-inflammatory activity in comparison to indomethacin (INM) as a reference drug. The results revealed that compound $8 g$ was the most active one exceeding the activity of the reference INM. Moreover, compounds $8 f$ and $8 k$ showed approximately the same results as INM after $5 \mathrm{hr}$. In silico ADME prediction investigations also forecasting the drug-like characters of these compounds .

\section{INTRODUCTION}

Coumarins (2H-1-benzopyran-2-ones) are important oxygen containing fused heterocycles used in preparation of pharmaceutical compounds and dyes ${ }^{1}$. They are a large class of lactones with a benzopyrone structure that have been isolated from plants as well as fully synthesized in the laboratory ${ }^{2}$. Natural coumarins have been shown antidiabetic activity ${ }^{3}$, anabolic, antioxidant and hepatoprotective activities ${ }^{4}$. Substituted coumarin derivatives have been reported to have variety of biological activities including anticoagulant $^{5}$, HIV protease inhibition ${ }^{6}$, CNS depressant $^{7}$, analgesic and anti-inflammatory activities $^{8,9}$. The potent antibiotics like novobiocin $^{10}, \quad$ coumarmycin $^{11} \quad$ and chlorobiocin $^{12}$ are coumarin derivatives. Among the various coumarin derivatives, 4methylcoumarin derivatives were present in various naturally occurring compounds which known to exhibit a wide range of biological and pharmaceutical activities ${ }^{13}$. Also, 7substituted coumarins are an important class of chemicals with a variety of bioactivities and uses $^{14}$. Moreover, 7-amino 4-methyl coumarin is also used as laser dye $\mathrm{e}^{15}$ and intermediate for the synthesis of bioactive compounds ${ }^{16}$.

Previous studies have shown that combining the coumarin backbone with some nitrogen-containing heterocyclic moieties could significantly broaden the spectrum of activity of these compounds and increase their anti- 
inflammatory, antimicrobial or anti-fungal efficiency $^{17-19}$.

Recent reports showed an amide moiety (i.e.-CONHAr) was thought to provide a better pharmacokinetic properties rather than an ester (i.e.- $\mathrm{OCOCH}_{3}$ ) moiety due to a higher chemical stability in both acidic and basic mediums. Chimenti et al., pointed out that, a carboxamides function in the coumarin ring might play an important role leading to various biological activities ${ }^{20}$. This prompted us to synthesize and study the anti-inflammatory activity of some new coumarin derivatives linked to amide groups to get the synergistic effect, Figure $1^{20}$.

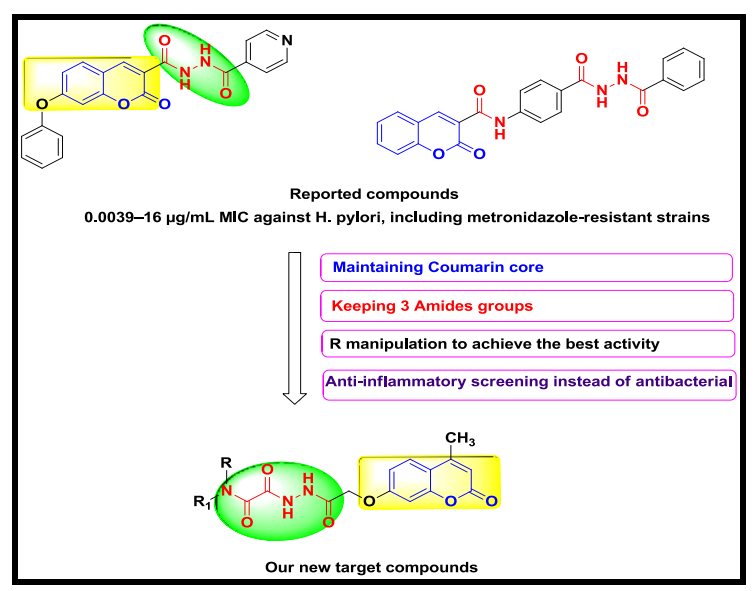

Fig. 1: Our design strategy

\section{MATERIALS AND METHODS}

\section{Chemistry}

Melting points were determined on an electro thermal melting point apparatus [Stuart Scientific, model SMP3, England, UK], and were uncorrected. A pre-coated silica gel plate (kieselgel $0.25 \mathrm{~mm}, \quad 60 \quad \mathrm{G} \quad \mathrm{F}_{254}$, Merck, Germany) was used for TLC monitoring of reactions. IR spectra ( $\mathrm{KBr}$ discs) were recorded on a Thermo Nicolet FT-IR spectrometer model 4700/6700 (Madison, USA) at faculty of science, Assiut University, Assiut, Egypt. ${ }^{1} \mathrm{H}-$ NMR spectra were scanned on a Varian EM360L NMR spectrophotometer $(60 \mathrm{MHz}$, Varian, USA) at Faculty of Pharmacy, JNMLA series FT-NMR system (400 MHz, JEOL, Tokyo, Japan) at Unit of Trace Analysis, Assiut University, Assiut, Egypt using DMSO$d_{6}$ as solvent. Chemical shifts are expressed in $\delta$-value (ppm) relative to TMS as internal standard, and deuterium oxide was used for the detection of exchangeable protons. Mass spectra were recorded with Gas Chromatography Mass, Quadruple-2010 Plus (Shimadzu, Kyoto, Japan) at the unit of Microanalysis, Faculty of Science, Cairo University, Elemental microanalyses were performed on a Vario elemental analyzer III (Vario, Hanau, Germany) at the unit of Microanalysis, Faculty of Science, Cairo University.

Reagents used for synthesis were purchased from Sigma-Aldrich and Merck. All solvents were obtained from commercial suppliers and used without further purification.

The starting materials 7-hydroxy-4-methyl coumarin $2^{21}$, ethyl 2-(4-methyl-2-oxo- $2 \mathrm{H}$ chromen-7-yloxy) acetate $3^{22}$, 2-(4-methyl-2oxo- $2 H$-chromen-7-yloxy) acetohydrazide $4^{23}$ were synthesized according to reported procedures.

\section{Synthesis of ethyl 2-(2-(2-((4-methyl-2-oxo- $2 H$-chromen-7-l)oxy)acetyl)hydrazinyl)-2- oxoacetate (5).}

To a suspension of (2-(4-methyl-2-oxo$2 \mathrm{H}$-chromen-7-yloxy)aceto-hydrazide (4) (2.48 $\mathrm{g}, 10 \mathrm{mmol})$ in anhydrous acetonitrile $(30 \mathrm{~mL})$, anhydrous pyridine $(1.2 \mathrm{~mL}, 15 \mathrm{mmol})$ was added. The suspension was stirred for $10 \mathrm{~min}$ at room temperature, and then cooled to $0^{\circ} \mathrm{C}$ before addition of ethyl oxalyl chloride $(1 \mathrm{~mL}$, $12 \mathrm{mmol}$ ) dropwise over approximately 15 mins. The reaction mixture was stirred at room temperature for $8 \mathrm{hrs}$ under nitrogen atmosphere, and then poured into ice cooled water. The separated yellowish white precipitate was filtered, washed with water, dried and crystallized from ethanol to afford compound (5). Yields, m.p. and elemental analyses are listed in Tables 1 while IR, ${ }^{1} \mathrm{H}$ NMR and mass spectral data are listed in Tables 2 .

Synthesis of 2-(2-(2-((4-methyl-2-oxo-2Hchromen-7-yl)oxy)acetyl)-hydrazinyl)-2oxoacetic acid (6).

To a suspension of ethyl 2-(2-(2-((4methyl-2-oxo-2 $H$-chromen-7-yl)oxy)acetyl)hydrazinyl)-2-oxoacetate (5) (3.48 g, 10 $\mathrm{mmol})$ in ethanol, $10 \%$ sodium hydroxide $(0.8$ $\mathrm{g}, 20 \mathrm{mmol}$ ) was added and the reaction mixture was stirred for $4 \mathrm{hrs}$ at $40^{\circ} \mathrm{C}$. After removal of the solvent under reduced pressure; the residue was dissolved in $\mathrm{H}_{2} \mathrm{O}$ and acidified 
with $2 \mathrm{~N} \mathrm{HCl}$. The formed precipitate was filtered, washed with $\mathrm{H}_{2} \mathrm{O}$ and dried to afford (6). Yields, m.p., and elemental analyses are listed in Table 1, while IR, ${ }^{1} \mathrm{H}$ NMR and mass spectral data are listed in Table 2.

\section{Synthesis of $\quad N$-substituted-2-(2-(2-((4- methyl-2-oxo-2H-chromen-7-yl)oxy)- acetyl)hydrazinyl)-2-oxoacetamide (8 a-o). General procedure}

To a suspension of 2-(2-(2-((4-methyl-2oxo-2H-chromen-7-yl)oxy)-acetyl)hydrazinyl)2-oxoacetic acid (6) (2 mmol) in dry DCM (10 $\mathrm{mL}$ ) with 2 drops of DMF, thionyl chloride (1 $\mathrm{mL}$ ) was add and the mixture refluxed for 3 hrs. The resulting suspension was evaporated to dryness under reduced pressure, and the residue of crude (7) was dispersed in DCM (10 $\mathrm{mL})$, then the solvent was eliminated under reduced pressure. Dispersion in DCM and solvent elimination was repeated twice...

To a suspension of the residue in dry DCM (10 $\mathrm{mL})$, appropriate amine $(2.4 \mathrm{mmol})$ was added and the reaction mixture was stirred for further $6 \mathrm{hr}$ at room temperature.

After removal of the DCM under reduced pressure, the residue was washed with $5 \% \mathrm{HCl}$ then with water repeatedly, dried over anhydrous $\mathrm{Na}_{2} \mathrm{SO}_{4}$ and concentrated under high vacuum. The obtained crude product was purified by column chromatography (60-120 mesh, 0.5//9.5 methanol/chloroform) to furnish the corresponding amides ( 8 a-o) in good to excellent yield. Yields, m.p., and elemental analyses are listed in Table 1 , while IR, ${ }^{1} \mathrm{H}$ NMR and mass spectral data are listed in Table2.

\section{Anti-inflammatory screening}

Male adult albino rats (120-150 g) were obtained from the animal house (Faculty of Medicine, Assiut University, Egypt). Indomethacin $\quad$ Liometacin $^{\circledR}$ vial, Nile company, Egypt), carrageenan (Sigma, USA), sodium carboxymethylcellulose (NaCMC) (El Nasr pharm. Company, Egypt) and normal saline (Almhenpharma company, Egypt).

Animals were housed in separate cages three animals each, in room temperature at $25 \pm 2^{\circ} \mathrm{C}$. Animals were allowed free access to rodent chow and water and maintained at a 12 hrs light/dark cycle. Work was conducted in accordance with the internationally accepted principles for laboratory animal's use and care as found in the European Community Guidelines ${ }^{24}$.

The test compounds and the reference drug were suspended in $1 \% \mathrm{NaCMC}$ in normal saline. Suspensions of the test compounds, reference drug and $1 \% \mathrm{NaCMC}$-saline solution (negative control) were injected i.p. $(1 \mathrm{~mL}$ each).

The anti-inflammatory activity of the test compounds was evaluated according to the carrageenan induced paw edema method ${ }^{25}$ in comparison to indomethacin as a reference drug. The test is based on pedal inflammation in rat paws induced by subplantar injection of carrageenan suspension $(0.2 \mathrm{~mL}$ of $1 \%$ solution in normal saline) into the right hind paw of the rats. Male adult albino rats were divided into groups of four animals each. The rat paw thickness was measured with a Vernier caliper (SMIEC, Shangahai, China) before and $1 \mathrm{hr}$ after carrageenan injection to detect the carrageenan induced inflammation. The test compounds and indomethacin, at a dose of 28 $\mu \mathrm{mol} / \mathrm{kg}$, were injected i.p. to all different groups of rats $1 \mathrm{hr}$ after carrageenan injection. In addition, a control group received the vehicle $1 \%$ NaCMC solution in normal saline. The difference between the thicknesses of the two paws was taken as a measure of edema inhibition. The measurement was carried out at $0.5,1,2,3,4$ and 5 hrs after injection of the test compounds, reference drug and control. The results are listed in Table 3.

The percentages of edema inhibition were calculated according to the following equation. $\%$ Edema $=\quad\left[\left(\mathrm{V}_{\mathrm{R}}-\mathrm{V}_{\mathrm{L}}\right) \quad\right.$ control $-\left(\mathrm{V}_{\mathrm{R}}-\mathrm{V}_{\mathrm{L}}\right)$ treated/( $\left.\mathrm{V}_{\mathrm{R}}-\mathrm{V}_{\mathrm{L}}\right)$ control $]_{\times} 100$

$\mathrm{V}_{\mathrm{R}}$ : Average right paw thickness, $\mathrm{V}_{\mathrm{L}}$ : Average left paw thickness. 
Table. 1: Physicochemical properties of compounds 5, 6 and 8 a-o.

\begin{tabular}{|c|c|c|c|c|c|c|}
\hline \multirow[t]{2}{*}{$\begin{array}{l}\text { Compd. } \\
\text { No. }\end{array}$} & \multirow[t]{2}{*}{ Yield (\%) } & \multirow[t]{2}{*}{ M.p. $\left({ }^{\circ} \mathrm{C}\right)$} & \multirow[t]{2}{*}{$\begin{array}{c}\text { M.F. } \\
\text { (M.Wt.) }\end{array}$} & \multicolumn{3}{|c|}{$\begin{array}{c}\text { Microanalyses } \\
\text { (calculated/found) }\end{array}$} \\
\hline & & & & $\mathrm{C} \%$ & $\mathrm{H} \%$ & $\mathrm{~N} \%$ \\
\hline 5 & $(80 \%)$ & $164-166^{\circ} \mathrm{C}$ & $\begin{array}{c}\mathrm{C}_{16} \mathrm{H}_{16} \mathrm{~N}_{2} \mathrm{O}_{7} \\
(348.31)\end{array}$ & $\begin{array}{l}55.17 \\
55.41\end{array}$ & $\begin{array}{l}4.63 \\
4.72 \\
\end{array}$ & $\begin{array}{l}8.04 \\
8.17 \\
\end{array}$ \\
\hline 6 & $(90 \%)$ & $113-116^{\circ} \mathrm{C}$ & $\begin{array}{c}\mathrm{C}_{14} \mathrm{H}_{12} \mathrm{~N}_{2} \mathrm{O}_{7} \\
(320.25)\end{array}$ & $\begin{array}{l}52.51 \\
52.74 \\
\end{array}$ & $\begin{array}{l}3.78 \\
3.81 \\
\end{array}$ & $\begin{array}{l}8.75 \\
8.89\end{array}$ \\
\hline $8 \mathrm{a}$ & $(65 \%)$ & $195-197^{\circ} \mathrm{C}$ & $\begin{array}{c}\mathrm{C}_{20} \mathrm{H}_{17} \mathrm{~N}_{3} \mathrm{O}_{6} \\
(395.37)\end{array}$ & $\begin{array}{l}60.76 \\
60.89 \\
\end{array}$ & $\begin{array}{l}4.33 \\
4.39 \\
\end{array}$ & $\begin{array}{l}10.63 \\
10.84 \\
\end{array}$ \\
\hline $8 \mathrm{~b}$ & $(62 \%)$ & $189-191^{\circ} \mathrm{C}$ & $\begin{array}{c}\mathrm{C}_{21} \mathrm{H}_{19} \mathrm{~N}_{3} \mathrm{O}_{6} \\
(409.39) \\
\end{array}$ & $\begin{array}{l}61.61 \\
61.94 \\
\end{array}$ & $\begin{array}{l}4.68 \\
4.73 \\
\end{array}$ & $\begin{array}{l}10.26 \\
10.34 \\
\end{array}$ \\
\hline $8 \mathrm{c}$ & $(72 \%)$ & $180-182^{\circ} \mathrm{C}$ & $\begin{array}{c}\mathrm{C}_{22} \mathrm{H}_{21} \mathrm{~N}_{3} \mathrm{O}_{6} \\
(423.42)\end{array}$ & $\begin{array}{l}62.41 \\
62.58\end{array}$ & $\begin{array}{l}5.00 \\
5.12\end{array}$ & $\begin{array}{c}9.92 \\
10.06\end{array}$ \\
\hline $8 \mathrm{~d}$ & $(60 \%)$ & $263-265^{\circ} \mathrm{C}$ & $\begin{array}{c}\mathrm{C}_{21} \mathrm{H}_{19} \mathrm{~N}_{3} \mathrm{O}_{6} \\
(409.39)\end{array}$ & $\begin{array}{l}61.61 \\
61.73\end{array}$ & $\begin{array}{l}4.68 \\
4.74\end{array}$ & $\begin{array}{l}10.26 \\
10.42\end{array}$ \\
\hline $8 \mathrm{e}$ & $(81 \%)$ & $221-223^{\circ} \mathrm{C}$ & $\begin{array}{c}\mathrm{C}_{21} \mathrm{H}_{19} \mathrm{~N}_{3} \mathrm{O}_{6} \\
(409.39) \\
\end{array}$ & $\begin{array}{l}61.61 \\
61.70 \\
\end{array}$ & $\begin{array}{l}4.68 \\
4.71 \\
\end{array}$ & $\begin{array}{l}10.26 \\
10.47 \\
\end{array}$ \\
\hline $8 \mathrm{f}$ & $(68 \%)$ & $241-243^{\circ} \mathrm{C}$ & $\begin{array}{c}\mathrm{C}_{21} \mathrm{H}_{19} \mathrm{~N}_{3} \mathrm{O}_{6} \\
(409.39)\end{array}$ & $\begin{array}{l}61.61 \\
61.76 \\
\end{array}$ & $\begin{array}{l}4.68 \\
4.73\end{array}$ & $\begin{array}{l}10.26 \\
10.44\end{array}$ \\
\hline $8 \mathrm{~g}$ & $(55 \%)$ & $179-181^{\circ} \mathrm{C}$ & $\begin{array}{c}\mathrm{C}_{21} \mathrm{H}_{19} \mathrm{~N}_{3} \mathrm{O}_{7} \\
(425.39)\end{array}$ & $\begin{array}{l}59.29 \\
59.43\end{array}$ & $\begin{array}{l}4.50 \\
4.55\end{array}$ & $\begin{array}{l}9.88 \\
9.95\end{array}$ \\
\hline $8 \mathrm{~h}$ & $(64 \%)$ & $186-188^{\circ} \mathrm{C}$ & $\begin{array}{c}\mathrm{C}_{21} \mathrm{H}_{19} \mathrm{~N}_{3} \mathrm{O}_{7} \\
(425.39) \\
\end{array}$ & $\begin{array}{l}59.29 \\
59.40 \\
\end{array}$ & $\begin{array}{l}4.50 \\
4.58 \\
\end{array}$ & $\begin{array}{r}9.88 \\
10.01 \\
\end{array}$ \\
\hline $8 \mathrm{i}$ & $(58 \%)$ & $215-217^{\circ} \mathrm{C}$ & $\begin{array}{c}\mathrm{C}_{20} \mathrm{H}_{16} \mathrm{FN}_{3} \mathrm{O}_{6} \\
(413.36)\end{array}$ & $\begin{array}{l}58.11 \\
58.34 \\
\end{array}$ & $\begin{array}{l}3.90 \\
3.97 \\
\end{array}$ & $\begin{array}{l}10.17 \\
10.32 \\
\end{array}$ \\
\hline $8 \mathrm{j}$ & $(63 \%)$ & $251-253^{\circ} \mathrm{C}$ & $\begin{array}{c}\mathrm{C}_{20} \mathrm{H}_{16} \mathrm{BrN}_{3} \mathrm{O}_{6} \\
(474.26)\end{array}$ & $\begin{array}{l}50.65 \\
50.81\end{array}$ & $\begin{array}{l}3.40 \\
3.42 \\
\end{array}$ & $\begin{array}{l}8.86 \\
8.94\end{array}$ \\
\hline $8 \mathrm{k}$ & $(59 \%)$ & $207-209^{\circ} \mathrm{C}$ & $\begin{array}{c}\mathrm{C}_{20} \mathrm{H}_{17} \mathrm{~N}_{3} \mathrm{O}_{7} \\
(411.36) \\
\end{array}$ & $\begin{array}{l}58.39 \\
58.52 \\
\end{array}$ & $\begin{array}{l}4.17 \\
4.23 \\
\end{array}$ & $\begin{array}{l}10.21 \\
10.43 \\
\end{array}$ \\
\hline 81 & $(72 \%)$ & $247-249^{\circ} \mathrm{C}$ & $\begin{array}{c}\mathrm{C}_{20} \mathrm{H}_{16} \mathrm{~N}_{4} \mathrm{O}_{8} \\
(440.36)\end{array}$ & $\begin{array}{l}54.55 \\
54.67\end{array}$ & $\begin{array}{l}3.66 \\
3.63 \\
\end{array}$ & $\begin{array}{l}12.72 \\
12.89 \\
\end{array}$ \\
\hline $8 \mathrm{~m}$ & $(79 \%)$ & $255-257^{\circ} \mathrm{C}$ & $\begin{array}{c}\mathrm{C}_{18} \mathrm{H}_{19} \mathrm{~N}_{3} \mathrm{O}_{6} \\
(373.36)\end{array}$ & $\begin{array}{l}57.90 \\
58.19 \\
\end{array}$ & $\begin{array}{l}5.13 \\
5.14 \\
\end{array}$ & $\begin{array}{l}11.25 \\
11.39\end{array}$ \\
\hline $8 \mathrm{n}$ & $(89 \%)$ & $260-261^{\circ} \mathrm{C}$ & $\begin{array}{c}\mathrm{C}_{19} \mathrm{H}_{21} \mathrm{~N}_{3} \mathrm{O}_{6} \\
(387.39)\end{array}$ & $\begin{array}{l}58.91 \\
59.12\end{array}$ & $\begin{array}{l}5.46 \\
5.52 \\
\end{array}$ & $\begin{array}{l}10.85 \\
11.01\end{array}$ \\
\hline 8 o & $(85 \%)$ & $249-251^{\circ} \mathrm{C}$ & $\begin{array}{c}\mathrm{C}_{18} \mathrm{H}_{19} \mathrm{~N}_{3} \mathrm{O}_{7} \\
(389.36)\end{array}$ & $\begin{array}{l}55.53 \\
55.81\end{array}$ & $\begin{array}{l}4.92 \\
4.95\end{array}$ & $\begin{array}{l}10.79 \\
10.94\end{array}$ \\
\hline
\end{tabular}


Table. 2: Spectral characterization of compounds 5, 6 and 8 a-o.

\begin{tabular}{|c|c|c|c|}
\hline $\begin{array}{c}\text { Compd. } \\
\text { No. }\end{array}$ & $\mathrm{IR}\left(\mathrm{KBr}, \mathrm{cm}^{-1}\right)$ & ${ }^{1} \mathrm{H}-\mathrm{NMR}$ ( DMSO- $d_{6}$ ) & $\operatorname{MS}(m / z)$ \\
\hline 5 & $\begin{array}{c}3467,3206(\mathrm{NH}) \\
1741,1712,1663 \\
(\mathrm{C}=\mathrm{O}), 1161(\mathrm{C}-\mathrm{O}- \\
\mathrm{C}) .\end{array}$ & $\begin{array}{c}\delta 1.29\left(\mathrm{t}, J=6.9 \mathrm{~Hz}, 3 \mathrm{H}, \mathrm{OCH}_{2} \mathrm{CH}_{3}\right), 2.41(\mathrm{~s}, \\
\left.3 \mathrm{H}, \mathrm{CH}_{3}\right), 4.29(\mathrm{q}, J=7.1 \mathrm{~Hz}, 2 \mathrm{H}, \\
\left.\mathrm{OC}_{2} \mathrm{CH}_{3}\right), 4.79\left(\mathrm{~s}, 2 \mathrm{H}, \mathrm{OCH}_{2}\right), 6.24(\mathrm{~s}, 1 \mathrm{H}, \\
\text { coumarin-H), } 7.00(\mathrm{~d}, J=2.4 \mathrm{~Hz}, 1 \mathrm{H}, \\
\text { coumarin-H), } 7.05(\mathrm{dd}, J=8.8,2.5 \mathrm{~Hz}, 1 \mathrm{H}, \\
\text { coumarin-H), } 7.73(\mathrm{~d}, J=8.8 \mathrm{~Hz}, 1 \mathrm{H}, \\
\text { coumarin-H), } 10.37\left(\mathrm{~s}, 1 \mathrm{H}, \mathrm{NHCOCH}, \mathrm{D}_{2} \mathrm{O}\right. \\
\text { exchangeable }), 10.84(\mathrm{~s}, 1 \mathrm{H}, \mathrm{COCON} \underline{\mathrm{H}}, \\
\left.\mathrm{D}_{2} \mathrm{O} \text { exchangeable }\right) .\end{array}$ & $\begin{array}{l}348.22\left(\mathrm{M}^{+}\right. \\
3.69 \%), 103.10 \\
(100 \%)\end{array}$ \\
\hline 6 & $\begin{array}{l}2700-3600(\mathrm{OH}) \\
1705,1663(\mathrm{C}=\mathrm{O}) \\
1160(\mathrm{C}-\mathrm{O}-\mathrm{C})\end{array}$ & $\begin{array}{c}\delta 2.4\left(\mathrm{~s}, 3 \mathrm{H}, \mathrm{CH}_{3}\right), 4.9\left(\mathrm{~s}, 2 \mathrm{H}, \mathrm{OCH}_{2}\right), 6.2(\mathrm{~s}, \\
1 \mathrm{H}, \text { coumarin- } \mathrm{H}), 7-7.3(\mathrm{~m}, 2 \mathrm{H}, \text { coumarin- } \\
\mathrm{H}), 7.6-7.8(\mathrm{~d}, 1 \mathrm{H}, \text { coumarin- } \mathrm{H}), 10.4(\mathrm{~s}, 2 \mathrm{H}, \\
\left.\text { CONHNHCO, exchangeable with } \mathrm{D}_{2} \mathrm{O}\right) \\
10.9\left(\mathrm{~s}, 1 \mathrm{H}, \mathrm{OH} \text {, exchangeable with } \mathrm{D}_{2} \mathrm{O}\right)\end{array}$ & - \\
\hline $8 \mathrm{a}$ & $\begin{array}{c}3272(\mathrm{NH}), 1728 \\
1671(\mathrm{C}=\mathrm{O}), 1447 \\
(\mathrm{C}=\mathrm{C} \text { Ar }), 700,748\end{array}$ & $\begin{array}{c}\delta 2.4\left(\mathrm{~s}, 3 \mathrm{H}, \mathrm{CH}_{3}\right), 5\left(\mathrm{~s}, 2 \mathrm{H}, \mathrm{OCH}_{2}\right), 6.4(\mathrm{~s} \\
1 \mathrm{H}, \text { coumarin-H), } 7.2-8(\mathrm{~m}, 8 \mathrm{H}, \mathrm{Ar}-\mathrm{H}), 10.4 \\
(\mathrm{~s}, 2 \mathrm{H}, \mathrm{CONHNHCO}), 10.6(\mathrm{~s}, 1 \mathrm{H}, \mathrm{Ph}-\mathrm{NH}- \\
\mathrm{CO}) .\end{array}$ & - \\
\hline $8 \mathrm{~b}$ & $\begin{array}{c}34990,3319,3262 \\
(\mathrm{NH}), 1714,1686, \\
1665(\mathrm{C}=\mathrm{O}), 1453 \\
(\mathrm{C}=\mathrm{C} \text { Ar }), 700 \\
748 .\end{array}$ & $\begin{array}{c}\delta 2.3\left(\mathrm{~s}, 3 \mathrm{H}, \mathrm{CH}_{3}\right), 4.3\left(\mathrm{~s}, 2 \mathrm{H}, \mathrm{NCH}_{2}\right), 4.8(\mathrm{~s}, \\
\left.2 \mathrm{H}, \mathrm{OCH}_{2}\right), 6.2(\mathrm{~s}, 1 \mathrm{H}, \text { coumarin-H), 6.9-7.1 } \\
(\mathrm{m}, 2 \mathrm{H}, \text { coumarin-H), 7.2-7.5 }(\mathrm{m}, 5 \mathrm{H}, \mathrm{Ar}-\mathrm{H}), \\
7.7(\mathrm{~d}, 1 \mathrm{H}, \text { coumarin-H), } 9.4(\mathrm{~s}, 1 \mathrm{H}, \\
\left.\mathrm{NHCH}_{2}\right), 10.3\left(\mathrm{~s}, 1 \mathrm{H}, \mathrm{CH}_{2}-\mathrm{CO}-\mathrm{NH}\right), 10.4(\mathrm{~s}, \\
1 \mathrm{H}, \mathrm{COCONHNH}) .\end{array}$ & - \\
\hline $8 \mathrm{c}$ & $\begin{array}{l}3260,3228,3170 \\
(\mathrm{NH}), 1704,1624 \\
(\mathrm{C}=\mathrm{O}), 1466(\mathrm{C}=\mathrm{C} \\
\text { Ar }), 746,698\end{array}$ & $\begin{array}{c}\delta 2.4\left(\mathrm{~s}, 3 \mathrm{H}, \mathrm{CH}_{3}\right), 2.81(\mathrm{t}, J=7.4 \mathrm{~Hz}, 2 \mathrm{H}, \\
\left.\mathrm{NHCH}_{2} \mathrm{CH}_{2}\right), 3.40(\mathrm{t}, J=5.5 \mathrm{~Hz}, 2 \mathrm{H}, \\
\left.\mathrm{NHCH}_{2} \mathrm{CH}_{2}\right), 4.76\left(\mathrm{~s}, 2 \mathrm{H}, \mathrm{OCH}_{2}\right), 6.25(\mathrm{~s}, \\
1 \mathrm{H}, \text { coumarin- } \mathrm{H}), 7.00(\mathrm{~d}, J=2.4 \mathrm{~Hz}, \\
1 \mathrm{H}, \text { coumarin-H), } 7.05(\mathrm{dd}, J=8.8,2.5 \mathrm{~Hz}, \\
1 \mathrm{H}, \text { coumarin- } \mathrm{H}), 7.18-7.33(\mathrm{~m}, 5 \mathrm{H}, \mathrm{Ar}-\mathrm{H}), \\
7.71(\mathrm{~d}, J=8.8 \mathrm{~Hz}, 1 \mathrm{H}, \text { coumarin-H), } 8.95 \\
\left(\mathrm{s}, 1 \mathrm{H}, \mathrm{CH}_{2} \mathrm{NHCO}_{\mathrm{H}} \mathrm{D}_{2} \mathrm{O} \text { exchangeable }\right), \\
10.29\left(\mathrm{~s}, 1 \mathrm{H}, \mathrm{NHCOCH}_{2}, \mathrm{D}_{2} \mathrm{O}\right. \\
\text { exchangeable }), 10.59(\mathrm{~s}, 1 \mathrm{H}, \mathrm{COCONHNH}, \\
\left.\mathrm{D}_{2} \mathrm{O} \text { exchangeable }\right) .\end{array}$ & $\begin{array}{c}423.26\left(\mathrm{M}^{+}\right. \\
0.86 \%), 275.19 \\
(100 \%)\end{array}$ \\
\hline $8 \mathrm{~d}$ & $\begin{array}{l}3428,3231(\mathrm{NH}) \\
2922(\mathrm{C}-\mathrm{H}), 1713 \\
1664(\mathrm{C}=\mathrm{O}), 1441 \\
(\mathrm{C}=\mathrm{C} \mathrm{Ar}), 746,700\end{array}$ & $\begin{array}{c}\delta 2.4\left(\mathrm{~s}, 3 \mathrm{H}, \mathrm{CH}_{3}\right), 3.4\left(\mathrm{~s}, 3 \mathrm{H}, \mathrm{CH}_{3} \mathrm{~N}\right), 4.8(\mathrm{~s}, \\
\left.2 \mathrm{H}, \mathrm{OCH}_{2}\right), 6.1(\mathrm{~s}, 1 \mathrm{H}, \text { coumarin-H), } 7.0- \\
7.2(\mathrm{~m}, 2 \mathrm{H}, \text { coumarin-H), 7.3-7.5 }(\mathrm{m}, 5 \mathrm{H}, \\
\text { Ar- } \mathrm{H}), 7.7(\mathrm{~d}, 1 \mathrm{H}, \text { coumarin-H), } 10.3(\mathrm{~s}, 2 \mathrm{H}, \\
\text { CONHNHCO }) .\end{array}$ & - \\
\hline 8 e & $\begin{array}{c}3292(\mathrm{NH}), 2918 \\
(\mathrm{C}-\mathrm{H}), 1693,1666 \\
(\mathrm{C}=\mathrm{O}), 1427(\mathrm{C}=\mathrm{C} \\
\text { Ar }), 817 .\end{array}$ & $\begin{array}{c}\delta 2.3\left(\mathrm{~s}, 3 \mathrm{H}, \mathrm{CH}_{3}\right), 2.4(\mathrm{~s}, 3 \mathrm{H}, \text { coumarin- } \\
\left.\mathrm{CH}_{3}\right), 4.8\left(\mathrm{~s}, 2 \mathrm{H}, \mathrm{OCH}_{2}\right), 6.3(\mathrm{~s}, 1 \mathrm{H}, \\
\text { coumarin-H), 7.1-7.8 (m, 7H, Ar-H), 10.5 (s, } \\
2 \mathrm{H}, \mathrm{CONHNHCO}), 10.8(\mathrm{~s}, 1 \mathrm{H}, \mathrm{CON} \underline{\mathrm{H}}-\mathrm{Ar}) .\end{array}$ & - \\
\hline $8 \mathrm{f}$ & $\begin{array}{c}3223(\mathrm{NH}), 2930 \\
(\mathrm{C}-\mathrm{H}), 1701,1670 \\
(\mathrm{C}=\mathrm{O}), 1450(\mathrm{C}=\mathrm{C} \\
\text { Ar }), 757 .\end{array}$ & $\begin{array}{c}\delta 2.2\left(\mathrm{~s}, 3 \mathrm{H}, \mathrm{CH}_{3}\right), 2.4\left(\mathrm{~s}, 3 \mathrm{H}, \mathrm{CH}_{3^{-}}\right. \\
\text {coumarin }), 4.8\left(\mathrm{~s}, 2 \mathrm{H}, \mathrm{OCH}_{2}\right), 6.3(\mathrm{~s}, 1 \mathrm{H}, \\
\text { coumarin-H), 7.1-7.8 (m, 7H, Ar-H), 10.3 }(\mathrm{s}, \\
2 \mathrm{H}, \mathrm{CONHNHCO}), 10.7(\mathrm{~s}, 1 \mathrm{H}, \mathrm{CON} \underline{\mathrm{H}}-\mathrm{Ar}) .\end{array}$ & \\
\hline
\end{tabular}


Table. 2: Continued

\begin{tabular}{|c|c|c|c|}
\hline $8 \mathrm{~g}$ & $\begin{array}{c}3292(\mathrm{NH}), 2994 \\
(\mathrm{C}-\mathrm{H}), 1697,1657 \\
(\mathrm{C}=\mathrm{O}), 1438(\mathrm{C}=\mathrm{C} \\
\mathrm{Ar}), 1143(\mathrm{C}-\mathrm{O}-\mathrm{C}), \\
831 .\end{array}$ & $\begin{array}{c}\delta 2.42\left(\mathrm{~s}, 3 \mathrm{H}, \mathrm{CH}_{3}\right), 3.75\left(\mathrm{~s}, 3 \mathrm{H}, \mathrm{OCH}_{3}\right), 4.80 \\
\left(\mathrm{~s}, 2 \mathrm{H}, \mathrm{OCH}_{2}\right), 6.25(\mathrm{~s}, 1 \mathrm{H}, \text { coumarin-H }), \\
6.94(\mathrm{~d}, J=2.4 \mathrm{~Hz}, 2 \mathrm{H}, \mathrm{Ar}-\mathrm{H}), 7.01(\mathrm{~d}, \mathrm{~J}=2.5 \\
\mathrm{Hz}, 1 \mathrm{H}, \text { coumarin-H), } 7.05(\mathrm{dd}, \mathrm{J}=8.8,2.5 \\
\mathrm{Hz}, 1 \mathrm{H}, \text { coumarin- } \mathrm{H}), 7.65-7.78(\mathrm{~m}, 3 \mathrm{H}, \mathrm{Ar}- \\
\mathrm{H}), 10.39\left(\mathrm{~s}, 1 \mathrm{H}, \mathrm{CH}_{2} \mathrm{CONH}, \mathrm{D}_{2} \mathrm{O}\right. \\
\text { exchangeable }), 10.68(\mathrm{~s}, 1 \mathrm{H}, \mathrm{COCONHNH}, \\
\left.\mathrm{D}_{2} \mathrm{O} \text { exchangeable }\right), 10.83(\mathrm{~s}, 1 \mathrm{H}, \mathrm{CONH}-\mathrm{Ar}, \\
\left.\mathrm{D}_{2} \mathrm{O} \text { exchangeable }\right)\end{array}$ & $\begin{array}{c}425.12\left(\mathrm{M}^{+},\right. \\
13.15 \%), 149.10 \\
(100 \%)\end{array}$ \\
\hline $8 \mathrm{~h}$ & $\begin{array}{c}3280,3217(\mathrm{NH}) \\
2928(\mathrm{C}-\mathrm{H}), 1730 \\
1635(\mathrm{C}=\mathrm{O}), 1463 \\
(\mathrm{C}=\mathrm{C} \text { Ar }), 1156 \\
(\mathrm{C}-\mathrm{O}-\mathrm{C}), 756\end{array}$ & $\begin{array}{c}\delta 2.4\left(\mathrm{~s}, 3 \mathrm{H}, \mathrm{CH}_{3}\right), 3.8\left(\mathrm{~s}, 3 \mathrm{H}, \mathrm{OCH}_{3}\right), 4.8(\mathrm{~s} \\
\left.2 \mathrm{H}, \mathrm{OCH}_{2}\right), 6.3(\mathrm{~s}, 1 \mathrm{H}, \mathrm{coumarin}-\mathrm{H}), 6.9-7.8 \\
(\mathrm{~m}, 7 \mathrm{H}, \mathrm{Ar}-\mathrm{H}), 9.8(\mathrm{~s}, 1 \mathrm{H}, \mathrm{CONH}-\mathrm{Ar}), 10.3 \\
(\mathrm{~s}, 2 \mathrm{H}, \mathrm{CONHNHCO}) .\end{array}$ & - \\
\hline $8 \mathrm{i}$ & $\begin{array}{c}3223(\mathrm{NH}), 1697 \\
1640(\mathrm{C}=\mathrm{O}), 1163 \\
(\mathrm{C}=\mathrm{C} \mathrm{Ar}), 835\end{array}$ & $\begin{array}{c}\delta 2.4\left(\mathrm{~s}, 3 \mathrm{H}, \mathrm{CH}_{3}\right), 5\left(\mathrm{~s}, 2 \mathrm{H}, \mathrm{OCH}_{2}\right), 6.3(\mathrm{~s}, \\
1 \mathrm{H}, \text { coumarin-H), 7.0-7.8 (m, 7H, Ar-H }), 10.3 \\
(\mathrm{~s}, 2 \mathrm{H}, \mathrm{CONHNHCO}), 10.5(\mathrm{~s}, 1 \mathrm{H}, \mathrm{CON} \underline{\mathrm{H}}- \\
\mathrm{Ar}) .\end{array}$ & - \\
\hline $8 \mathrm{j}$ & $\begin{array}{c}3280,(\mathrm{NH}), 1697 \\
1650(\mathrm{C}=\mathrm{O}), 1437 \\
(\mathrm{C}=\mathrm{C} \text { Ar }), 826\end{array}$ & $\begin{array}{c}\delta 2.4\left(\mathrm{~s}, 3 \mathrm{H}, \mathrm{CH}_{3}\right), 4.8\left(\mathrm{~s}, 2 \mathrm{H}, \mathrm{OCH}_{2}\right), 6.1(\mathrm{~s}, \\
1 \mathrm{H}, \text { coumarin-H), 6.9-7.8 (m, 7H, Ar-H }), 10.2 \\
(\mathrm{~s}, 1 \mathrm{H}, \mathrm{CON} \underline{\mathrm{H}}-\mathrm{Ar}), 10.4(\mathrm{~s}, 2 \mathrm{H}, \\
\text { CONHNHCO }) .\end{array}$ & - \\
\hline $8 \mathrm{k}$ & $\begin{array}{c}3400-3100(\mathrm{OH}) \\
3219(\mathrm{NH}), 1713 \\
1658(\mathrm{C}=\mathrm{O}), 1428 \\
(\mathrm{C}=\mathrm{C} \mathrm{Ar}), 1078 \\
(\mathrm{C}-\mathrm{O}), 843\end{array}$ & $\begin{array}{c}\delta 2.4\left(\mathrm{~s}, 3 \mathrm{H}, \mathrm{CH}_{3}\right), 5\left(\mathrm{~s}, 2 \mathrm{H}, \mathrm{OCH}_{2}\right), 6.2(\mathrm{~s}, \\
1 \mathrm{H}, \text { coumarin-H), 6.9-7.8 }(\mathrm{m}, 7 \mathrm{H}, \mathrm{Ar}-\mathrm{H}), 10.3 \\
(\mathrm{~s}, 2 \mathrm{H}, \mathrm{CONHNHCO}), 10.5(\mathrm{~s}, 1 \mathrm{H}, \mathrm{CONH}- \\
\mathrm{Ar}), 11.4(\mathrm{~s}, 1 \mathrm{H}, \mathrm{OH}) .\end{array}$ & - \\
\hline 81 & $\begin{array}{c}3481,3360(\mathrm{NH}) \\
1709,1655(\mathrm{C}=\mathrm{O}) \\
1505,1391\left(\mathrm{NO}_{2}\right) \\
1445(\mathrm{C}=\mathrm{C} \mathrm{Ar}) \\
756 .\end{array}$ & $\begin{array}{c}\delta 2.4\left(\mathrm{~s}, 3 \mathrm{H}, \mathrm{CH}_{3}\right), 4.7\left(\mathrm{~s}, 2 \mathrm{H}, \mathrm{OCH}_{2}\right), 6.2(\mathrm{~s}, \\
1 \mathrm{H}, \text { coumarin-H), 6.9-7.8 (m, 7H, Ar-H }), 10.3 \\
(\mathrm{~s}, 2 \mathrm{H}, \mathrm{CONHNHCO}), 11.1(\mathrm{~s}, 1 \mathrm{H}, \mathrm{CONH}- \\
\mathrm{Ar}) .\end{array}$ & - \\
\hline $8 \mathrm{~m}$ & $\begin{array}{l}3487,3233(\mathrm{NH}) \\
2978(\mathrm{C}-\mathrm{H}), 1704 \\
1644(\mathrm{C}=\mathrm{O}), 1080 \\
(\mathrm{C}-\mathrm{N})\end{array}$ & $\begin{array}{c}\delta 1.85\left(\mathrm{tq}, J=13.1,6.6 \mathrm{~Hz}, 4 \mathrm{H},\left(\mathrm{CH}_{2}\right)_{2}\right), 2.41 \\
\left(\mathrm{~s}, 3 \mathrm{H}, \mathrm{CH}_{3}\right), 3.31\left(\mathrm{~m}, 4 \mathrm{H}, \mathrm{N}\left(\mathrm{CH}_{2}\right)_{2}\right), 4.77(\mathrm{~s}, \\
\left.2 \mathrm{H}, \mathrm{OCH}_{2}\right), 6.25(\mathrm{~s}, 1 \mathrm{H}, \text { coumarin- } \mathrm{H}), 7.01 \\
(\mathrm{~d}, J=2.4 \mathrm{~Hz}, 1 \mathrm{H}, \text { coumarin-H }), 7.05(\mathrm{dd}, J= \\
8.8,2.4 \mathrm{~Hz}, 1 \mathrm{H}, \text { coumarin- } \mathrm{H}), 7.73(\mathrm{~d}, J=8.8 \\
\mathrm{Hz}, 1 \mathrm{H}, \text { coumarin-H}), 10.30(\mathrm{~s}, 1 \mathrm{H}, \\
\left.\mathrm{NHCOCH}, \mathrm{D}_{2} \mathrm{O} \text { exchangeable }\right), 10.53(\mathrm{~s}, \\
\left.1 \mathrm{H}, \mathrm{COCONH}_{1} \mathrm{D}_{2} \mathrm{O} \text { exchangeable }\right)\end{array}$ & $\begin{array}{c}373.44\left(\mathrm{M}^{+}\right. \\
0.90 \%), 98.09 \\
(100 \%) .\end{array}$ \\
\hline $8 \mathrm{n}$ & $\begin{array}{l}3269(\mathrm{NH}), 2951 \\
(\mathrm{C}-\mathrm{H}), 1720,1660 \\
(\mathrm{C}=\mathrm{O}), 1079(\mathrm{C}- \\
\mathrm{N})\end{array}$ & $\begin{array}{c}\text { 81.3-1.6 }\left(\mathrm{m}, 6 \mathrm{H},\left(\mathrm{CH}_{2}\right)_{3}\right), 2.3\left(\mathrm{~s}, 3 \mathrm{H}, \mathrm{CH}_{3}\right) \\
\text { 3.3-3.6 }\left(\mathrm{m}, 4 \mathrm{H}, \mathrm{N}\left(\mathrm{CH}_{2}\right)_{2}\right), 4.6\left(\mathrm{~s}, 2 \mathrm{H}, \mathrm{OCH}_{2}\right) \\
\text { 6.1 }(\mathrm{s}, 1 \mathrm{H}, \text { coumarin- } \mathrm{H}), 6.8-7.1(\mathrm{~m}, 2 \mathrm{H} \\
\text { coumarin-H), 7.6 }(\mathrm{d}, 1 \mathrm{H}, \text { coumarin- } \mathrm{H}), 10.3 \\
(\mathrm{~s}, 2 \mathrm{H}, \mathrm{CONHNHCO}) .\end{array}$ & - \\
\hline 80 & $\begin{array}{l}3259(\mathrm{NH}), 2926 \\
(\mathrm{C}-\mathrm{H}), 1716,1666 \\
(\mathrm{C}=\mathrm{O}), 1114(\mathrm{C}-\mathrm{O}- \\
\mathrm{C}), 1079(\mathrm{C}-\mathrm{N})\end{array}$ & $\begin{array}{c}\delta 2.4\left(\mathrm{~s}, 3 \mathrm{H}, \mathrm{CH}_{3}\right), 3.2-3.8(\mathrm{~m}, 8 \mathrm{H}, \\
\text { morpholine-H), } 4.7\left(\mathrm{~s}, 2 \mathrm{H}, \mathrm{OCH}_{2}\right), 6.1(\mathrm{~s}, 1 \mathrm{H}, \\
\text { coumarin-H), 6.9-7.1 (m, } 2 \mathrm{H}, \text { coumarin-H), } \\
7.6(\mathrm{~d}, 1 \mathrm{H}, \text { coumarin-H), } 10.3(\mathrm{~s}, 2 \mathrm{H}, \\
\text { CONHNHCO }) .\end{array}$ & - \\
\hline
\end{tabular}

(-): Not determined 


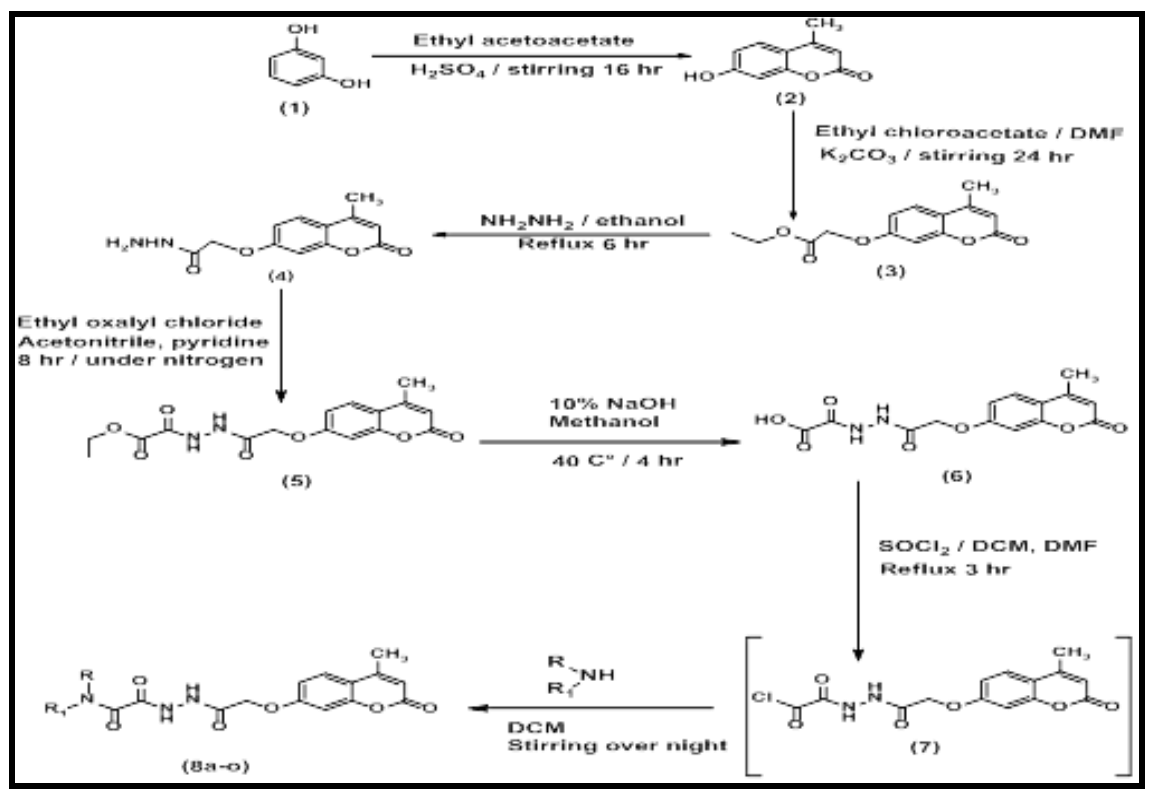

\begin{tabular}{|c|c|c|c|c|c|}
\hline Comp. No. & $\mathrm{R}$ & $\mathrm{R}_{1}$ & Comp. No. & $\mathrm{R}$ & $\mathrm{R}_{1}$ \\
\hline $8 \mathrm{a}$ & $\mathrm{H}$ & $\mathrm{C}_{6} \mathrm{H}_{5}$ & $8 \mathrm{i}$ & $\mathrm{H}$ & $p-\mathrm{F}-\mathrm{C}_{6} \mathrm{H}_{4}$ \\
\hline $8 \mathrm{~b}$ & $\mathrm{H}$ & $\mathrm{CH}_{2}-\mathrm{C}_{6} \mathrm{H}_{5}$ & $8 \mathrm{j}$ & $\mathrm{H}$ & $p-\mathrm{Br}-\mathrm{C}_{6} \mathrm{H}_{4}$ \\
\hline $8 \mathrm{c}$ & $\mathrm{H}$ & $\mathrm{CH}_{2} \mathrm{CH}_{2}-\mathrm{C}_{6} \mathrm{H}_{5}$ & $8 \mathrm{k}$ & $\mathrm{H}$ & $p-\mathrm{OH}-\mathrm{C}_{6} \mathrm{H}_{4}$ \\
\hline $8 \mathrm{~d}$ & $\mathrm{CH}_{3}$ & $\mathrm{C}_{6} \mathrm{H}_{5}$ & 81 & $\mathrm{H}$ & $p-\mathrm{NO}_{2}-\mathrm{C}_{6} \mathrm{H}_{4}$ \\
\hline $8 \mathrm{e}$ & $\mathrm{H}$ & $p-\mathrm{CH}_{3}-\mathrm{C}_{6} \mathrm{H}_{4}$ & $8 \mathrm{~m}$ & & yrrolidine \\
\hline $8 \mathrm{f}$ & $\mathrm{H}$ & $o-\mathrm{CH}_{3}-\mathrm{C}_{6} \mathrm{H}_{4}$ & $8 n$ & & iperidine \\
\hline $8 \mathrm{~g}$ & $\mathrm{H}$ & $p-\mathrm{OCH}_{3}-\mathrm{C}_{6} \mathrm{H}_{4}$ & 80 & & orpholine \\
\hline $8 \mathrm{~h}$ & $\mathrm{H}$ & $o-\mathrm{OCH}_{3}-\mathrm{C}_{6} \mathrm{H}_{4}$ & & & \\
\hline
\end{tabular}

Scheme 1: Synthetic route of compounds 8a-o

\section{RESULTS AND DISCUSSION}

\section{Chemistry}

The starting 2-(4-methyl-2-oxo- $2 \mathrm{H}$ chromen-7-yloxy) acetohydrazide 4 was prepared by refluxing ethyl 2-((4-methyl-2oxo- $2 \mathrm{H}$-chromen-7-yl)oxy) acetate 3 with hydrazine hydrate in ethanol ${ }^{23}$. Structure of compound 4 was confirmed by comparison its physical and spectral data with the reported ones ${ }^{9}$.
The new intermediate, compound 5 was prepared by treating compound 4 with ethyl oxalyl chloride using acetonitrile as solvent in presence of pyridine to afford ethyl 2-(2-(2-((4methyl-2-oxo-2H-chromen-7yl)oxy)acetyl) hydrazinyl)-2-oxoacetate 5 in an excellent yield.

IR spectrum of compound 5 showed two bands at 3467 and $3206 \mathrm{~cm}^{-1}$ for two $(\mathrm{NH})$ groups and one band at $1161 \mathrm{~cm}^{-1}$ for (C-O-C), in addition to three bands at 1741, 1712 and $1663 \mathrm{~cm}^{-1}$ represent $(\mathrm{C}=\mathrm{O})$ groups. 
${ }^{1} \mathrm{H}$ NMR spectrum of compound 5 displayed a triplet signal at $\delta 1.29 \mathrm{ppm}$ and quartet at $\delta 4.29 \mathrm{ppm}$ corresponding to ethyl group. Moreover, the spectrum illustrated two singlet signals at $\delta 10.36$ and $10.80 \mathrm{ppm}$ which exchangeable with $\mathrm{D}_{2} \mathrm{O}$ represent both $(\mathrm{NH})$, in addition to characteristic patterns of coumarin aromatic protons. The structure of 5 has been also confirmed from EI-MS, the spectrum showed the molecular ion peak $\mathbf{M}^{+}$at $\mathrm{m} / z$ $348.22(3.69 \%)$ corresponding to its relative molecular mass (348.10) and a base peak at $\mathrm{m} / \mathrm{z}$ $103.10(100 \%)$. It is further confirmed by elemental analysis.

Hydrolysis of compound 5 using 10\% $\mathrm{NaOH}$ in methanol afford another new intermediate 2-(2-(2-((4-methyl-2-oxo- $2 \mathrm{H}$ chromen-7-yl)oxy)acetyl)hydrazinyl)-2oxoacetic acid 6 in $90 \%$ yield.

IR spectrum of 6 characterized by a broad band at $2700-3600 \mathrm{~cm}^{-1}$ for acidic $(\mathrm{OH})$, and a strong absorption band at $1705 \mathrm{~cm}^{-1}$ corresponding to $(\mathrm{C}=\mathrm{O})$ carboxylic.

Moreover, ${ }^{1} \mathrm{H}$ NMR data also confirmed the hydrolysis of ester; disappearance of ethyl group characteristic protons, instead new signal for acidic $(\mathrm{OH})$ appeared which a convincing evidence of acid formation.

Refluxing compound 6 with thionyl chloride in DCM for $3 \mathrm{hrs}$ afforded the acid chloride 7 which used directly in the next step without further purification due its sensitivity against light and moisture. The acid chloride 7 was stirred overnight with an appropriate amine to afford the corresponding carboxamides 8 a-o.

IR spectra of the new compounds 8 a-o displayed no absorption bands belonging to $(\mathrm{OH})$ group, instead new bands for aryl substitutions, reappearance of $(\mathrm{NH})$ bands at $3233-3487 \mathrm{~cm}^{-1}$, in addition to bands of $\mathrm{C}=\mathrm{O}$ groups at $1720-1644 \mathrm{~cm}^{-1}$.

${ }^{1} \mathrm{H}$ NMR spectra also confirmed the disappearance of $\mathrm{OH}$ signal and displaying an exchangeable signal around $\delta 10 \mathrm{ppm}$ for $\mathrm{NH}$ protons in addition to the introduced of aromatic moiety protons at $\delta 6.8-8 \mathrm{ppm}$.

As a representative example, ${ }^{1} \mathrm{H}$ NMR spectrum of compound $8 \mathrm{c}$ showed two characteristic triplet signals at $\delta 2.81$ and 3.40 ppm corresponding to $\mathrm{CH}_{2} \mathrm{CH}_{2} \mathrm{~N}$, a multiplet signal at $\delta$ 7.18-7.33 ppm integrated for aromatic $5 \mathrm{H}$, in addition to the signals of the original nucleus.

Moreover, EI-MS of $8 \mathrm{c}$ showed a weak molecular ion peak $\mathrm{M}^{+}$at $\mathrm{m} / \mathrm{z} 423.26(0.86 \%)$ corresponding to its relative molecular mass (423.14) and a base peak at $\mathrm{m} / \mathrm{z} 275.19$ (100\%).

In another example, ${ }^{1} \mathrm{H}$ NMR data also confirm compound $8 \mathrm{~g}$ structure, it showed two singlets at $\delta 3.75$ and $4.80 \mathrm{ppm}$ corresponding to $\mathrm{OCH}_{3}$ and $\mathrm{OCH}_{2}$ respectively, a pair of doublets at $\delta 7.01$ and $7.70 \mathrm{ppm}$ integrating for four protons corresponding to $p$-disubstituted benzene moiety, and three amidic protons at $\delta$ $10.39,10.68$ and $10.83 \mathrm{ppm}$ exchangeable with $\mathrm{D}_{2} \mathrm{O}$

Furthermore, EI-MS analysis also confirms the suggested structures of compound $8 \mathrm{~g}$. The spectrum showed a molecular ion peak $\mathrm{M}^{+}$at $m / z 425.12$ (13.15\%) corresponding to its relative molecular mass (425.12) and a base peak at $\mathrm{m} / z 149.10(100 \%)$ which approved the structure.

\section{Anti-inflammatory activity}

Results of anti-inflammatory activities of compounds 5, 6, 8 a-o, Table 3, showed gradual increasing in activity up to maximum 4-5 h except compound $8 \mathrm{c}$ and $8 \mathrm{~m}$ which showed their maximum effects after $3 \mathrm{hrs}$.

Intermediates 5 and 6 displayed moderate to good activities relative to INM as they showed 59 and $76 \%$ of the anti-inflammatory of INM respectively after $4 \mathrm{hrs}$, while they showed 63 and $70 \%$ respectively after $5 \mathrm{hrs}$.

Compounds 8 a, 8 b, 81 and 8 o showed as significance activity achieving $84-94 \%$ of indomethacin activity at different time interval. Also, compound $8 \mathrm{~g}$ exceeding the activity of INM at $5 \mathrm{hrs}$ interval; achieving 113\% comparing with the reference drug. Moreover, compounds $8 \mathrm{f}$ and $8 \mathrm{k}$ showed approximately the same results as INM after $5 \mathrm{hrs}$. 
Table. 3: Percentage of edema inhibition of compounds 5, 6, 8 a-o and indomethacin.

\begin{tabular}{|c|c|c|c|c|c|c|}
\hline \multirow{2}{*}{$\begin{array}{c}\text { Comp. } \\
\text { No. }\end{array}$} & \multicolumn{6}{|c|}{ Percentage of edema inhibition $\pm \mathrm{SE}$} \\
\hline & 30 mins & $1 \mathrm{hr}$ & $2 \mathrm{hr}$ & $3 \mathrm{hr}$ & $4 \mathrm{hr}$ & $5 \mathrm{hr}$ \\
\hline Control & - & - & - & - & - & - \\
\hline IND & $22.22 \pm 0.29$ & $33.33 \pm 0.22$ & $67.39 \pm 0.23$ & $78.72 \pm 0.14$ & $78.72 \pm 0.20$ & $79.16 \pm 0.22$ \\
\hline 5 & $4.88 \pm 0.25$ & $14.66 \pm 0.14$ & $17.39 \pm 1.10$ & $27.65 \pm 0.45$ & $46.17 \pm 0.22$ & $49.79 \pm 0.14$ \\
\hline 6 & $13.35 \pm 0.36$ & $20.68 \pm 0.15$ & $30.43 \pm .018$ & $51.06 \pm 0.65$ & $59.57 \pm 0.20$ & $55.20 \pm 0.72$ \\
\hline $8 \mathrm{a}$ & $18.01 \pm 0.54$ & $23.78 \pm 0.24$ & $36.73 \pm 1.05$ & $31.48 \pm 0.25$ & $66.59 \pm 0.28$ & $60.41 \pm 0.84$ \\
\hline $8 \mathrm{~b}$ & $22.44 \pm 0.54$ & $29.11 \pm 0.65$ & $57.82 \pm 0.45$ & $67.02 \pm 0.65$ & $74.46 \pm 0.84$ & $54.16 \pm 0.28$ \\
\hline $8 \mathrm{c}$ & $11.56 \pm 0.78$ & $21.12 \pm 0.74$ & $56.53 \pm 0.65$ & $56.59 \pm 0.46$ & $46.59 \pm 0.75$ & $47.91 \pm 0.29$ \\
\hline $8 \mathrm{~d}$ & $22.66 \pm 0.28$ & $28.23 \pm 0.54$ & $58.70 \pm 1.23$ & $59.57 \pm 0.15$ & $66.17 \pm 0.42$ & $54.16 \pm 0.14$ \\
\hline $8 \mathrm{e}$ & $22.00 \pm 0.47$ & $30.18 \pm 0.68$ & $40.21 \pm 1.36$ & $54.25 \pm 0.35$ & $61.70 \pm 0.13$ & $58.33 \pm 0.29$ \\
\hline $8 \mathrm{f}$ & $20.88 \pm 0.69$ & $28.89 \pm 0.14$ & $41.30 \pm 0.47$ & $57.44 \pm 0.95$ & $68.08 \pm 0.16$ & $79.16 \pm 0.29$ \\
\hline $8 \mathrm{~g}$ & $29.33 \pm 0.96$ & $52.12 \pm 0.27$ & $57.17 \pm 0.63$ & $63.19 \pm 0.76$ & $83.19 \pm 0.54$ & $89.16 \pm 1.20$ \\
\hline $8 \mathrm{~h}$ & $29.36 \pm 1.25$ & $22.15 \pm 0.22$ & $19.34 \pm 0.54$ & $21.27 \pm 0.81$ & $24.46 \pm 0.47$ & $25.12 \pm 0.25$ \\
\hline $8 \mathrm{i}$ & $1.56 \pm 0.57$ & $17.72 \pm 0.22$ & $22.60 \pm 0.03$ & $20.00 \pm 0.73$ & $51.06 \pm 0.96$ & $50.00 \pm 0.67$ \\
\hline $8 \mathrm{j}$ & $8.22 \pm 1.06$ & $16.14 \pm 0.26$ & $16.73 \pm 0.17$ & $41.48 \pm 0.84$ & $42.76 \pm 0.54$ & $58.33 \pm 0.61$ \\
\hline $8 \mathrm{k}$ & $28.73 \pm 1.04$ & $57.30 \pm 0.24$ & $61.95 \pm 0.62$ & $70.20 \pm 0.15$ & $78.72 \pm 0.69$ & $79.17 \pm 0.36$ \\
\hline 81 & $17.87 \pm 0.23$ & $45.07 \pm 0.34$ & $56.08 \pm 0.48$ & $58.93 \pm 0.12$ & $71.27 \pm 0.47$ & $75.83 \pm 0.46$ \\
\hline $8 \mathrm{~m}$ & $19.43 \pm 0.38$ & $36.67 \pm 0.16$ & $52.82 \pm 0.74$ & $63.82 \pm 0.13$ & $60.85 \pm 0.52$ & $60.41 \pm 0.94$ \\
\hline $8 \mathrm{n}$ & $16.47 \pm 0.27$ & $27.67 \pm 0.84$ & $49.78 \pm 1.7$ & $60.42 \pm 0.52$ & $67.65 \pm 0.82$ & $67.29 \pm 0.31$ \\
\hline 80 & $24.23 \pm 0.65$ & $25.00 \pm 0.78$ & $48.04 \pm 0.65$ & $54.04 \pm 0.17$ & $74.47 \pm 0.93$ & $46.66 \pm 0.18$ \\
\hline
\end{tabular}

Significant difference from control group at $\mathrm{p}<0.05$

Moreover, by comparing the activities of different $N$-substitutions; compounds 8 a-o, the results revealed that the anti-inflammatory activity of compound $8 \mathrm{~g}\left(\mathrm{R}=\mathrm{H}, \mathrm{R}_{1}=p-\mathrm{OCH}_{3}\right)$ higher than that of other derivatives where $\mathrm{R}_{1}=$ $p-\mathrm{CH}_{3}, p-\mathrm{NO}_{2}, p-\mathrm{F}, p-\mathrm{Br}, p-\mathrm{OH}$, Figure 2.

Also, in comparing the anti-inflammatory activities of compounds $8 \mathrm{~m}, 8 \mathrm{n}$ and $8 \mathrm{o}$ with different saturated heterocyclic substitutions, their results showed that 8 o with morpholine moiety was the most active one with $95 \%$ activity comparable to INM after 4 hrs, Figure3.

\section{Structure activity relationship}

Presence of substituted-phenyl part directly attached to amide moiety is seemed to enhance the anti-inflammatory activity than unsubstituted phenyl or benzyl group using 


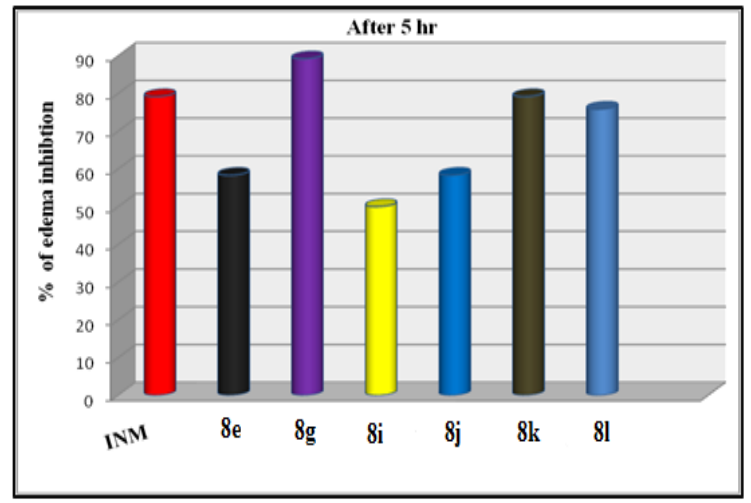

Fig. 2: Anti-inflammatory activity of compounds 8 e, $8 \mathrm{~g}, 8 \mathrm{i}, 8 \mathrm{j}, 8 \mathrm{k}, 8 \mathrm{l}$ and INM after $5 \mathrm{hr}$

Moreover, para-substitutions on the phenyl ring had proven a much greater antiinflammatory effect over the $o$-substitutions; especially with oxygen containing functionalities, either electron donating groups (eg. hydroxyl or methoxy) or electron withdrawing groups (eg. $\mathrm{NO}_{2}$ ).

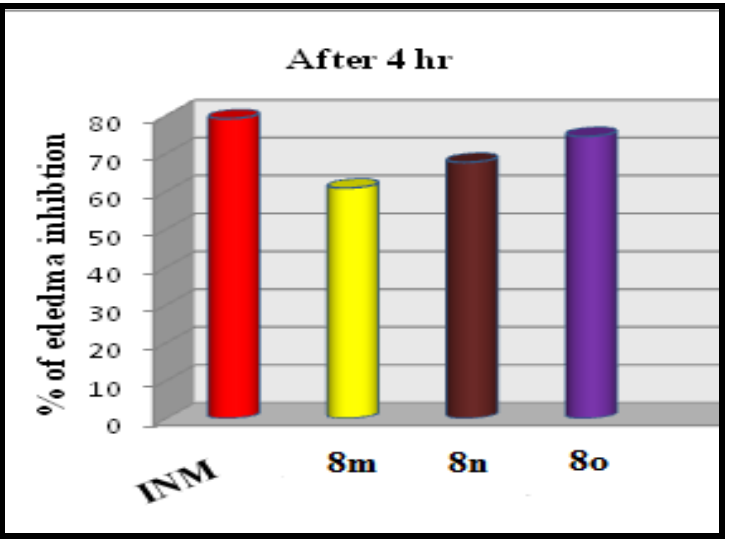

Fig. 3: Anti-inflammatory activity of $8 \mathrm{~m}, 8 \mathrm{n}, 8 \mathrm{o}$ and INM

\section{Computational analysis In-silico forecasting of physicochemical properties, pharmacokinetic profile, and drug-likeness profile}

Clinical trials of new drugs are known to be extremely difficult due to improper ADME (absorption, delivery, metabolism, and excretion) properties, as well as the high costs of developing a new drug. As a result, determining the pharmacokinetic properties of a new drug is an important phase in the drug development process that can help focus lead optimization efforts on recovered analogues ${ }^{26}$. In silico $\mathrm{ADME}$ screens can now be used to identify the most promising compounds and reduce the possibility of late-stage drug attrition $^{27}$. To achieve a desirable in-vivo response, pharmacodynamic and pharmacokinetic properties must be in balance. Predictions of delivery volume, brain penetration, oral bioavailability, and clearance also provide more information about the regimen and medication dose ${ }^{28}$. A variety of parameters are investigated using virtual screening methods, including partition coefficient, drug likeness score, polar surface area PSA, human intestinal absorption HIA, and cell permeability. If the molecular weight is less than 500, $\log \mathrm{P}$ is less than 5, the number of hydrogen bond acceptors is less than 10 , and the number of hydrogen bond donors is less than 5 , an available orally drug is chosen in accordance with Lipinski's law $^{29}$. The number of rotatable bonds is used to denote molecular stability, which is essential for oral bioavailability; if the molecule is flexible, it means the drug is not as effective when taken orally. Also, since polar surface area (PSA) is inversely proportional to percentage absorption (percent ABS), it has been suggested that the amount of hydrogen bonding groups be replaced with polar surface area (PSA) as a component and involved in the measurement of percentage absorption (percent ABS).

$$
\% \mathrm{ABS}=109-0.345 \mathrm{PSA}
$$

Oral bioavailability should be strong for compounds with a PSA of less than 140 A2 and 10 or less rotatable bonds ${ }^{30}$.

For predicting the pharmacokinetic parameters of the most active compounds, we used the Molsoft ${ }^{31}$, Pre-ADMET ${ }^{32}$, SwissADME $^{33}$, and Molinspiration ${ }^{34}$ softwares.

From the results, compounds stratify according to Lipinski's law, with MW ranging from 389.36 to $425.39(<500)$, HBD ranging from 2 to $4(<5), \log \mathrm{P}$ values ranging from 0.52 to $1.78(<5)$, and HBA ranging from 6 to 7 (<10). As a result, they should have good oral absorption, and variations in bioactivity cannot be attributed to this property. Moreover, the compounds had numbers of rotatable bonds ranging from 8 to $9(<10)$ and topological PSA values of 126.74 and 146.97 A2 (<140 A2), respectively, suggesting good permeability, absorption, and transport across biological membranes.

In addition, the drug-likeness model score for compounds was calculated using Molsoft software (Table 5). Aqueous solubility can 
affect absorption and distribution characteristics. In this case, these compounds met the requirements of the drug-likeness model. The higher the drug-likeness model ranking, the more likely it is to be a drug molecule. Compound 8 o (0.24) was predicted to have a positive model-score, while the other compounds were predicted to have a negative model-score (-0.09 to -0.40$)$.

Pre-ADMET software was also used to conduct an in silico analysis of the following pharmacokinetic parameters: Human intestinal absorption (HIA), Caco2 (human colon adenocarcinoma) permeability coefficient, inhibition of cytochrome P4502D6 (CYP2D6), MDCK (Madin-Darby canine kidney cells) permeability coefficient, Blood brain barrier partition coefficient (BBB), and human plasma protein binding (PPB). The estimated ADME parameters' results are shown in the table below (Table 6). The findings showed that the compounds had a medium CNS absorption range of 0.2 to $0.4(0.1-2)$; In the $\mathrm{CaCo}-2$ and MDCK models, the investigated compounds had cell permeability ranging from 2.90 to $18.21 \mathrm{~nm} / \mathrm{s}$ and 0.36 to $30.85 \mathrm{~nm} / \mathrm{s}$, respectively. They were also non-inhibitors of the CYP2D6 enzyme, implying that they would have no interactions with CYP2D6 inhibitors and/or inducers.

Consequently, they showed strong human intestinal absorption values ranging from 80.00 to $90.25 \%$ ( $\leq 80 \%)$, suggesting very wellabsorbed compounds, the examined compounds were found to be low bound to human plasma proteins, ranging from 40.32 to 81.83 percent $(\geq 90 \%)$.

Table. 4 : Physicochemical and lipophilicity of the potent compounds using SwissADME and Molinspiration

\begin{tabular}{|c|c|c|c|c|c|c|c|c|c|c|}
\hline \multirow[b]{2}{*}{ Code } & \multirow{2}{*}{$\begin{array}{c}\text { Lipophilici } \\
\text { ty } \\
\text { Consensus } \\
\log \mathrm{P}\end{array}$} & \multicolumn{9}{|c|}{ Physicochemical properties } \\
\hline & & $\begin{array}{l}\mathrm{MW}^{\mathrm{a}} \\
\mathrm{g} / \mathrm{mol}\end{array}$ & $\begin{array}{l}\text { Heavy } \\
\text { atoms }\end{array}$ & $\begin{array}{c}\text { Aromatic } \\
\text { heavy } \\
\text { atoms }\end{array}$ & $\begin{array}{c}\text { Rotatable } \\
\text { Bonds }\end{array}$ & $\begin{array}{l}\text { H-bond } \\
\text { acceptors }\end{array}$ & $\begin{array}{c}\text { H-bond } \\
\text { donors }\end{array}$ & $\mathrm{MR}^{\mathrm{b}}$ & $\begin{array}{c}\text { TPSA }^{\mathrm{c}} \\
\left(\mathrm{A}^{2}\right)\end{array}$ & $\begin{array}{c}\% \\
\text { ABS }^{\text {d }}\end{array}$ \\
\hline $8 \mathbf{a}$ & 1.68 & 395.37 & 29 & 16 & 9 & 6 & 3 & 103.39 & 126.74 & 65.27 \\
\hline 8 b & 1.77 & 409.39 & 30 & 16 & 9 & 6 & 3 & 106.66 & 126.74 & 65.27 \\
\hline $8 \mathrm{i}$ & 2.03 & 413.36 & 30 & 16 & 9 & 7 & 3 & 103.35 & 126.74 & 65.27 \\
\hline 80 & 0.52 & 389.36 & 28 & 10 & 8 & 7 & 2 & 99.58 & 127.18 & 65.12 \\
\hline $8 \mathrm{f}$ & 1.78 & 425.39 & 31 & 16 & 9 & 7 & 3 & 109.89 & 135.97 & 62.09 \\
\hline $8 \mathrm{k}$ & 1.33 & 411.36 & 30 & 16 & 9 & 7 & 4 & 105.42 & 146.97 & 58.29 \\
\hline
\end{tabular}

Abbreviation: ${ }^{\mathrm{a}} \mathrm{MW}$, molecular weight; ${ }^{\mathrm{b}} \mathrm{MR}$, molar refractivity; ${ }^{\mathrm{c}} \mathrm{TPSA}$, topological polar surface area; ${ }^{\mathrm{d}} \% \mathrm{ABS}$ : percentage of absorption

Table. 5: Lipinski drug likeness of the most active compounds using Molsoft and wissADME software.

\begin{tabular}{|c|c|c|c|}
\hline Code & Drug likeness model score & $\begin{array}{c}\text { Lipinski } \\
\text { violations }\end{array}$ & Bioavailability score \\
\hline $8 \mathrm{a}$ & -0.38 & 0 & 0.55 \\
\hline $8 \mathrm{~b}$ & -0.18 & 0 & 0.55 \\
\hline $8 \mathrm{i}$ & -0.09 & 0 & 0.55 \\
\hline $8 \mathrm{o}$ & 0.24 & 0 & 0.55 \\
\hline $8 \mathrm{f}$ & -0.40 & 0 & 0.55 \\
\hline $8 \mathrm{k}$ & -0.15 & 0 & 0.55 \\
\hline
\end{tabular}


Table. 6: ADME data of most active compounds calculated using preADMET software

\begin{tabular}{|c|c|c|c|c|c|c|}
\hline \multirow{2}{*}{ Code } & \multicolumn{7}{|c|}{ Pharmacokinetics $^{\mathrm{n}}$} \\
\cline { 2 - 7 } & BBB $^{\mathrm{a}}$ & Caco-2 $^{\mathrm{b}}$ & HIA $^{\mathrm{c}}$ & MDCK $^{\mathrm{d}}$ & PPB $^{\mathrm{e}}$ & CYP 2D6 $^{\mathrm{f}}$ \\
\hline $8 \mathrm{a}$ & 0.30 & 9.93 & 89.71 & 16.59 & 79.28 & None \\
\hline $8 \mathrm{~b}$ & 0.30 & 9.58 & 90.25 & 30.85 & 81.83 & None \\
\hline $8 \mathrm{i}$ & 0.30 & 18.21 & 89.75 & 0.36 & 80.55 & None \\
\hline $8 \mathrm{o}$ & 0.40 & 2.90 & 84.23 & 11.08 & 40.32 & None \\
\hline $8 \mathrm{f}$ & 0.20 & 14.62 & 88.85 & 0.58 & 72.16 & None \\
\hline $8 \mathrm{k}$ & 0.40 & 15.13 & 80.00 & 0.38 & 71.41 & None \\
\hline
\end{tabular}

${ }^{\mathrm{a}} \mathrm{BBB}$ : blood brain barrier penetration; ${ }^{\mathrm{b}} \mathrm{CACO}-2$ : permeability through cells derived from human colon adenocarcinoma; ${ }^{\circ} \mathrm{HIA}$ : percentage human intestinal absorption; ${ }^{\mathrm{d}} \mathrm{MDCK}$ : permeability through Madin-Darby canine kidney cells; ${ }^{\mathrm{e} P P B}$ : plasma protein binding; ${ }^{\mathrm{f}} \mathrm{CYP} 2 \mathrm{D} 6$ : cytochrome P450 2D6

\section{Conclusion}

In summary, a new series of coumarinyloxyacetamide derivatives with three synergistic amide groups was designed, synthesized in good yields, and structurally elucidated using IR, ${ }^{1} \mathrm{HNMR}$ and mass spectrometry along with elemental microanalyses. All new finals and intermediate compounds were tested for their possible antiinflammatory activities using in-vivo carrageenan induced paw edema method and they showed a significance activity especially compound $8 \mathrm{~g}\left(\mathrm{R}=\mathrm{H}, \mathrm{R}_{1}=p-\mathrm{OCH}_{3}\right)$ which exceed the activity of the reference drug achieving $113 \%$ activity of indomethacin. Also compounds $8 \mathrm{f}\left(\mathrm{R}=\mathrm{H}, \mathrm{R}_{1}=o-\mathrm{CH}_{3}\right)$ and $8 \mathrm{k}$ $\left(\mathrm{R}=\mathrm{H}, \mathrm{R}_{1}=p-\mathrm{OH}\right)$ showed approximately the same results as indomethacin. Moreover, among different saturated heterocyclic substitutions; compound 8 o with morpholine moiety was the most active one with $95 \%$ activity comparable to indomethacin.

\section{REFERENCES}

1. X.-y. Sun, T. Liu, J. Sun, and X.-j. Wang, "Synthesis and application of coumarin fluorescence probes," RSC Advances, 10 (18), 10826-10847 (2020).

2. M. Lončarić, D. Gašo-Sokač, S. Jokić, and M. Molnar, "Recent advances in the synthesis of coumarin derivatives from different starting materials", Biomolecules, 10 (1), 151 (2020).

3. H. Li, Y. Yao, and L. Li, "Coumarins as potential antidiabetic agents," Journal of Pharmacy and Pharmacology, 69 (10), 1253-1264 (2017).

4. X.-M. Peng, G. LV Damu, and H. Zhou, "Current developments of coumarin compounds in medicinal chemistry", Current pharmaceutical design, 19 (21), 3884-3930 (2013).

5. N. C. Bang, A. Abyshev, and D. Y. Ivkin, "Synthesis and in vivo evaluation of new coumarin conjugates as potential indirect-action anticoagulants", Pharmaceutical Chemistry Journal, 53 (5), 419-422 (2019).

6. S. Mishra, A. Pandey, and S. Manvati, "Coumarin: An emerging antiviral agent", Heliyon, 6 (1), e03217 (2020).

7. S.-B. Wang, H. Liu, G.-Y. Li, J. Li, X.-J. Li, K. Lei, L.-C. Wei, Z.-S. Quan, X.-K. Wang, and R.-M. Liu, "Coumarin and 3, 4-dihydroquinolinone derivatives: Synthesis, antidepressant activity, and molecular docking studies", Pharmacological Reports, 71 (6), 12441252 (2019).

8. Y. Bansal, P. Sethi, and G. Bansal, "Coumarin: A potential nucleus for antiinflammatory molecules," Medicinal Chemistry Research, 22 (7), 3049-3060 (2013).

9. M. A. Elbastawesy, B. G. Youssif, M. H. Abdelrahman, and A. M. Hayallah, "Synthesis and biological evaluation of some new coumarin derivatives as potential antimicrobial, analgesic and anti-inflammatory agents", Der Pharma Chemica, 7 (10), 337-349 (2015).

10. D. Ramirez, L. Berry, R. Domalaon, M. Brizuela, and F. Schweizer, "Dilipid 
Ultrashort Tetrabasic Peptidomimetics Potentiate Novobiocin and Rifampicin Against Multidrug-Resistant GramNegative Bacteria", ACS infectious diseases, 6 (6), 1413-1426 (2020).

11. K. Shiozawa, M. Oka, H. Soda, M. Yoshikawa, Y. Ikegami, J. Tsurutani, K. Nakatomi, Y. Nakamura, S. Doi, and T. Kitazaki, "Reversal of breast cancer resistance protein (BCRP/ABCG2)-mediated drug resistance by novobiocin, a coumermycin antibiotic", International journal of cancer, 108 (1), 146-151 (2004).

12. M. G. Marcu, T. W. Schulte, and L. Neckers, "Novobiocin and related coumarins and depletion of heat shock protein 90-dependent signaling proteins", Journal of the National Cancer Institute, 92 (3), 242-248 (2000).

13. F. G. Medina, J. G. Marrero, M. MacíasAlonso, M. C. González, I. CórdovaGuerrero, A. G. T. García, and S. Osegueda-Robles, "Coumarin heterocyclic derivatives: Chemical synthesis and biological activity", Natural product reports, 32 (10), 14721507 (2015).

14. M. Asif, "Pharmacologically potentials of different substituted coumarin derivatives", Chemistry International 1 (1), 1-11 (2015).

15. K. Żamojć, W. Wiczk, B. Zaborowski, D. Jacewicz, and L. Chmurzyński, "Fluorescence quenching of 7-amino-4methylcoumarin by different TEMPO derivatives", Spectrochimica Acta Part A: Molecular and Biomolecular Spectroscopy, 136, 1875-1880 (2015).

16. R. Tandon, P. Ponnan, N. Aggarwal, R. Pathak, A. S. Baghel, G. Gupta, A. Arya, M. Nath, V. S. Parmar, and H. G. Raj, "Characterization of 7-amino-4methylcoumarin as an effective antitubercular agent: Structure-activity relationships", Journal of antimicrobial chemotherapy, 66 (11), 2543-2555 (2011).

17. M. Raghu, A. Nagaraj, and C. S. Reddy, "Synthesis and in vitro study of novel bis-[3-(2-arylmethylidenimino-1,

3-thiazol-4-yl)-4-hydroxy-2H-chromen-2 -one-6-yl] methane and bis-[3-(2-arylidenhydrazo-1,

3-thiazol-4-yl)-4-hydroxy-2H-chromen-2 -one-6-yl] methane as potential antimicrobial agents", Journal of Heterocyclic Chemistry, 46 (2), 261-267 (2009).
18. P. M. Ronad, M. N. Noolvi, S. Sapkal, S. Dharbhamulla, and V. S. Maddi, "Synthesis and antimicrobial activity of 7-(2-substituted phenylthiazolidinyl)benzopyran-2-one derivatives", European journal of medicinal chemistry, 45 (1), 85-89 (2010).

19. R. S. Keri, K. M. Hosamani, R. V. Shingalapur, and H. R. Seetharama Reddy, "RETRACTED: 2-Azetidinone derivatives: Design, synthesis, in vitro anti-microbial, cytotoxic activities and DNA cleavage study", European Journal of Medicinal Chemistry, 44 (12), 5123-5130 (2009).

20. F. Chimenti, B. Bizzarri, A. Bolasco, D. Secci, P. Chimenti, S. Carradori, A. Granese, D. Rivanera, D. Lilli, and A. Zicari, "A novel class of selective antiHelicobacter pylori agents 2-oxo-2Hchromene-3-carboxamide derivatives", Bioorganic \& medicinal chemistry letters, 17 (11), 3065-3071 (2007).

21. P. Arora, S. Das, N. Arora, A. Gawai, and U. Baghel, "Synthesis and screening of some novel 7-Hydroxy 4-Methyl Coumarin derivatives for antipsychotic activity", International Journal of Pharmacy \& Life Sciences, 1, 113-118 (2010).

22. B. G. M. Youssif, "Synthesis and biological evaluation of some new coumarin derivatives as antimicrobial agents," Bulletin of Pharmaceutical Sciences. Assiut, 36 (2), 105-116 (2013).

23. D. P. Kardile, M. R. Holam, A. S. Patel, and S. B. Ramani, "Synthesis and biological evaluation of (7-hydroxy-2oxo-2H-chromen-4-Yl) acetic acid hydrazide derivatives used as a potent biological agents", Rasayan Journal of Chemistry, 4 (1), 66-72 (2011).

24. P. K. Chow, R. T. Ng, and B. E. Ogden, Using animal models in biomedical research: A primer for the investigator. (World Scientific, 2008).

25. L. Nargund, G. Reddy, and V. Hariprasad, "Anti-inflammatory activity of substituted 1, 3, 4-oxadiazoles", Journal of pharmaceutical sciences, 83 (2), 246-248 (1994).

26. N. Ö. Can, D. Osmaniye, S. Levent, B. N. Sağlık, B. Korkut, Ö. Atlı, Y. Özkay, and Z. A. Kaplancikl1, "Design, synthesis and biological assessment of new thiazolylhydrazine derivatives as selective and reversible hMAO-A inhibitors", European journal of medicinal chemistry, 144, 68-81 (2018).

27. F. Cheng, W. Li, Y. Zhou, J. Shen, Z. $\mathrm{Wu}, \mathrm{G}$. Liu, P. W. Lee, and Y. Tang, 
"Correction to "admetSAR: A comprehensive source and free tool for assessment of chemical ADMET properties"", Journal of chemical information and modeling, 59 (11), 4959-4959 (2019).

28. H. Van De Waterbeemd and E. Gifford, "ADMET in silico modelling: Towards prediction paradise", Nature reviews Drug discovery, 2 (3), 192-204 (2003).

29. M. M. Morcoss, M. El Shimaa, R. A. Ibrahem, H. M. Abdel-Rahman, M. Abdel-Aziz, and D. A. Abou El-Ella, "Design, synthesis, mechanistic studies and in silico ADME predictions of benzimidazole derivatives as novel antifungal agents", Bioorganic Chemistry, 101, 103956 (2020).

30. D. F. Veber, S. R. Johnson, H.-Y. Cheng, B. R. Smith, K. W. Ward, and K. D. Kopple, "Molecular properties that influence the oral bioavailability of drug candidates", Journal of medicinal chemistry, 45 (12), 2615-2623 (2002).
31. https://www.molsoft.com/.

32. S. Bioinformatics and Molecular Design Research Center, South Corea, PreADMET program, 2004. Available from: URL: http://preadmet.bmdrc.org.

33. A. Daina, O. Michielin, and V. Zoete, "SwissADME: a free web tool to evaluate pharmacokinetics, drug-likeness and medicinal chemistry friendliness of small molecules", Scientific reports, 7 (1), 1-13 (2017).

34. S. G. Molinspiration Cheminformatics, Slovak Republic, Molinspiration Program, 2002. Available from: URL: http://www.molinspiration.com. 


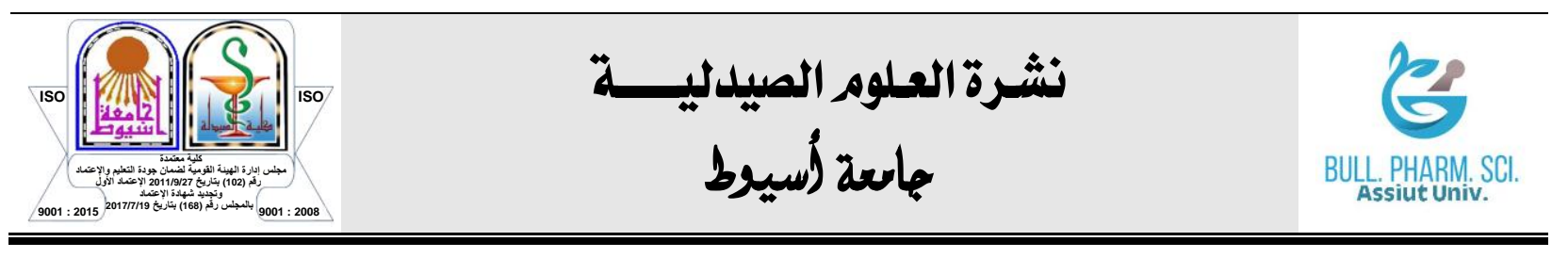

تخليق ودراسة الخواص الفيزيوكيميائية والتأثير البيولوجى لبعض مشتثات البهات

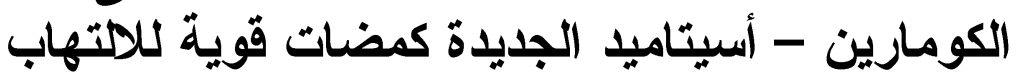

محمد عبد الرحمن ابر اهيم البسطويسي' - مرثا محب مرقس ' - مصطفى حامد عبد الرحمن' - بهاء

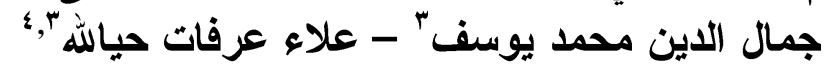

'قسم الكيمياء العضوية الصيدلية ، كلية الصيدلة ، جامعة الأزهر فرع أسيوط ، عبهالV ، مصر

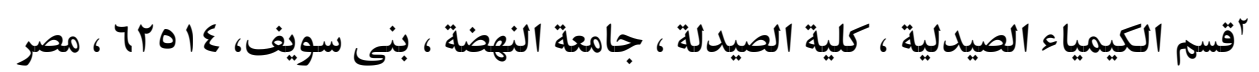

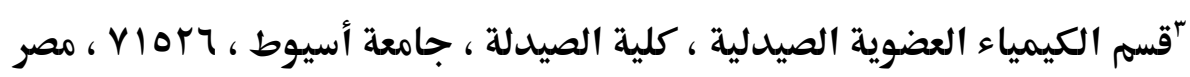

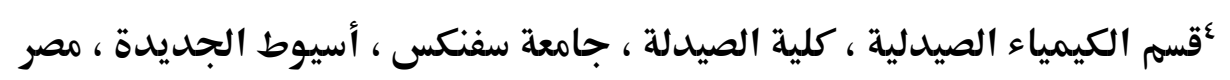

تم عمل مجمو عة جديدة من مشتقات الميثيل كومارين مرتبطة بثلاث مجمو عات فعالة من جزئ

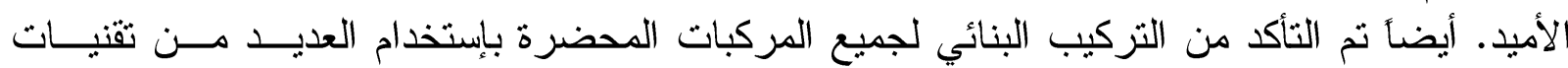

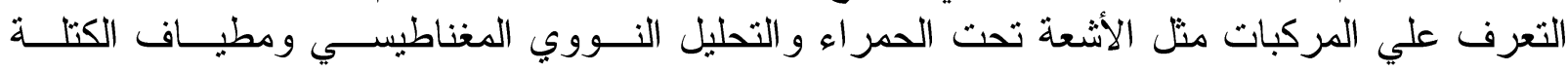

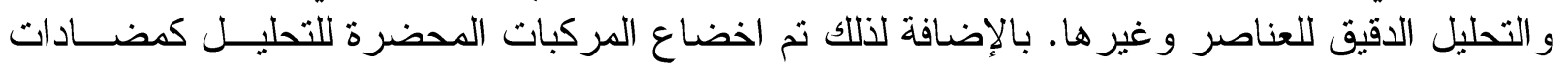

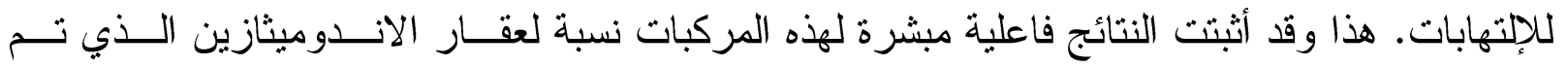

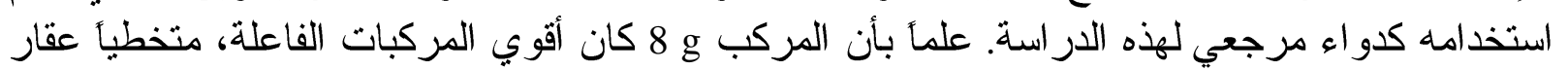

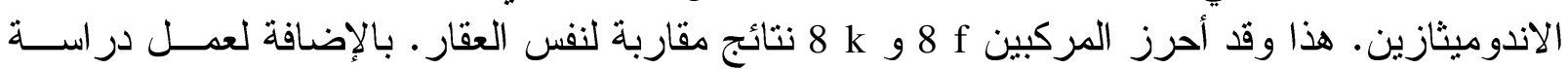

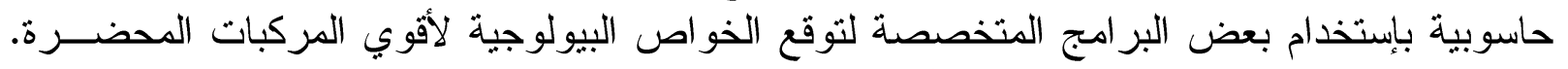
توطئة لإحتمالية استخدام هذه المركبات كأدوية. 